\title{
Vaccine by Chicken Line Interaction Alters the Protective Efficacy against Challenge with a Very Virulent plus Strain of Marek's Disease Virus in White Leghorn Chickens
}

\author{
Shuang Chang ${ }^{1,2}$, John R. Dunn ${ }^{1}$, Mohammad Heidari ${ }^{1}$, Lucy F. Lee ${ }^{1}$, Catherine W. Ernst ${ }^{2}$, \\ Jiuzhou Song ${ }^{3}$, Huanmin Zhang ${ }^{1 \#}$ \\ ${ }^{1}$ Avian Disease and Oncology Laboratory, Agriculture Research Service, USDA, East Lansing, USA; ${ }^{2}$ Department of Animal Sci- \\ ence, Michigan State University, East Lansing, USA; ${ }^{3}$ Department of Animal and Avian Sciences, University of Maryland, College \\ Park, USA. \\ Email: "Huanmin.zhang@ars.usda.gov
}

Received September $27^{\text {th }}, 2011$; revised October $31^{\text {st }}, 2011$; accepted November $14^{\text {th }}, 2011$

\begin{abstract}
Marek's disease (MD) is a lymphoproliferative disease of domestic chickens caused by Marek's disease virus (MDV), an oncogenic and highly contagious $\alpha$-herpesvirus. MD has been controlled by vaccination but sporadic outbreaks of MD still occur in some parts of the world. Efforts to improve vaccine efficacy have continued in both research communities and vaccine industries. We reported the host genetic variation affecting Marek's disease vaccine-induced immunity in chickens earlier. In this study, we evaluated chicken lines, vaccines, and line by vaccine interaction on the protective efficacy of vaccination against MD. Specific pathogen free chickens from the relatively resistant line $6_{3}$ and the highly susceptible line $7_{2}$ were primarily used to evaluate the protection by three kinds of vaccines ( $\mathrm{rMd} 5 \Delta \mathrm{Meq}$, CVI988/Rispens, and HVT) upon challenge with a very virulent plus strain of MDV, vv+648A. Our data confirmed that both the chicken line and the vaccine significantly affected the protective efficacy of vaccination and showed that a chicken line by vaccine interaction, in most of the trials, also altered vaccine protective efficacy. More interestingly, although the protective index of all vaccine strains was higher in resistant than in susceptible line of chickens, the difference for HVT protection was striking and warrants further study. The findings may have important implications for vaccine development as well as for selective use of particular vaccines in specific lines of chickens to achieve maximum protection at minimized costs.
\end{abstract}

Keywords: Marek’s Disease Vaccine; Vaccine Efficacy; Host Genetics; Vaccine by Chicken Line Interaction

\section{Introduction}

Marek's disease (MD) is an acute viral neoplastic disease in chickens, caused by a cell-associated $\alpha$-herpesvirus of the genus Mardivirus, commonly referred to as Marek's disease virus (MDV). MD is characterized by the presence of $T$ cell lymphoma as well as infiltration of nerves and organs by lymphocytes [1]. There are three serotypes of MDV based on their antigenic differences and pathogenicity. Serotype 1 is an oncogenic and pathogenic virus causing MD in susceptible chickens. Serotypes 2 (e.g. SB-1) and 3 (turkey herpesvirus, HVT) are apathogenic $[2,3]$. MD remains a significant problem for the poultry industry throughout the world due to losses from con-

\footnotetext{
"The work conducted by Drs. Zhang, Dunn, Heidari, and Lee as US Government Employees cannot be copyrighted.

${ }^{*}$ Corresponding author.
}

demnation and cost of vaccination [4].

Vaccines have been used to control MD from the 1970s [5]. Since MD sporadically occurs world-wide and MDV is capable of survival for an extended long period of time both in birds and in the surrounding environment of infected poultry houses, the control of MD essentially depends on proper implementation of strategies that ensure successful use of vaccines [6,7]. Commonly used MD vaccines are derived from different serotypes of MDV. The commercial MD vaccine that is currently used and considered to be the most effective is CVI988/Rispens derived from serotype $1 \mathrm{MDV}$ [8]. SB-1 and HVT are the commonly used serotype 2 and serotype $3 \mathrm{MD}$ vaccines, respectively [9]. The serotype 1 and 2 vaccines are used only in cell-associated form. The serotype 3 HVT can be in both cell-associated and cell-free forms. 
The cell-associated HVT has been more widely used than its cell-free form since it is less expensive to produce and is more protective against MD in chickens that are maternal antibody positive [10], as most chicks are in commercial settings. MD vaccine prevents the formation of lymphoma and other MD symptoms in susceptible chickens. It does not prevent MDV infection, replication, or horizontal spread [6].

In addition to factors such as type of vaccines, vaccine dosage, virulence and dose of challenge virus, interval between vaccination and MDV challenge that affect vaccine efficacy [4,11], host genetics also plays an important role in vaccine protective efficacy. In 1979, Gavora and Spencer [12] reported that MD incidences of some chicken lines were altered by vaccination. Using experimental lines of 15.B-congenic chickens, Bacon and Witter [13-16] then reported that chickens with major histocompatibility complex $(M H C) B^{*} 15$ haplotype developed better protection against $\mathrm{MD}$ than those with $B * 2$ or $B^{*} 13$ haplotypes after receiving vaccine of either serotype 1,2 , or 3 . In addition, chickens with $B * 5$ or $B * 21$ developed variable protection after vaccination with different $\mathrm{MD}$ vaccines of serotypes 1,2 , and 3 . It was further concluded that the $B$-haplotype influence on vaccinal immunity against MD is significant in both experimental strains and commercial lines of chickens, and that MD vaccines should be selectively used based on the predominant $B$-haplotype(s) of chicken flocks to maximize vaccine protection against MD. Using experimental lines of a series of recombinant congenic strains and the highly inbred progenitor lines $6_{3}$ and $7_{2}$, which all share the same $M H C B * 2$ haplotype, non- $M H C$ genetics effect on vaccine efficacy was suggested in our recent report [17].

The lines $6_{3}$ and $7_{2}$ are highly inbred specific pathogen free (SPF) White Leghorn chickens developed and maintained at the Avian Disease and Oncology Laboratory (ADOL). Although lines $6_{3}$ and $7_{2}$ share a common $M H C$, they differ in non-MHC genetic variation (based on DNA fingerprinting using a $3 \mathrm{~K}$ single nucleotide polymorphism panel [18] (Zhang et al., unpublished data). Other relevant genetic differences between the lines $6_{3}$ and $7_{2}$ include : resistance to MD [19], lymphoid organ size [20], and immunoglobulin level [21]. The present study was designed to assess the effect of chicken line, vaccine, and line by vaccine interaction on vaccine protective efficacy against $\mathrm{MD}$ induced by a very virulent plus $\left(\mathrm{vv}^{+}\right)$strain of MDV, 648A, in resistant and susceptible lines of chickens.

\section{Materials and Methods}

\subsection{Lines of Chickens}

SPF chickens from two highly inbred lines $\left(6_{3}\right.$, MD re- sistant; 72, MD susceptible) were used in trials 1 and 2 . In addition to these two inbred lines, highly MD susceptible $15 \mathrm{I}_{5} \times 7_{1}$ hybrid chickens (crossbred between the line $15 \mathrm{I}_{5}$ and line $7_{1}$ ) were also sampled once in trial 3 to compare with the lines $6_{3}$ and $7_{2}$. The lines $6_{3}$ and $7_{2}$ share a common $M H C B * 2$ haplotype, whereas $15 \mathrm{I}_{5} \times 7_{1}$ chickens are heterozygous for $B * 2 / B^{*} 15$ [22,23]. Due to a management policy change, all chickens used in the first trial were maternal antibody positive for HVT, but the chickens of trials 2 and 3 were all maternal antibody negative. All experimental chickens were managed during and euthanized at the end of the experiments closely following the ADOL's Guidelines for Animal Care and Use (Revised April, 2005) and the Guidelines for Care and Use of Laboratory Animals published by Institute for Laboratory Animal Research (ILAR Guide) in 1996 (http://www.nap.edu/openbook.php?record_id=5140).

\subsection{Virus and Vaccines}

The $\mathrm{vv}^{+}$strain of Marek's disease virus, 648A [24,25], was used to challenge the vaccinated or unvaccinated experimental chickens. Three MD vaccines were used in trials 1 and 2, which included a candidate MD vaccine, rMd5 $\Delta$ Meq [26], a commercial CVI988/Rispens MD vaccine (CVI988/Rispens 1), and the cell-associated HVT FC126 (ADOL HVT) $[27,28]$. Both rMd5 $\Delta$ Meq and HVT FC126 were propagated in SPF chicken embryo fibroblast. Additional commercial CVI988/Rispens (CVI988/ Rispens 2) and HVT (commercial HVT) from different manufacturers were included in trial 3.

\subsection{Experiment Design}

Randomly sampled chicks from each of the lines were divided into vaccinal treatment groups in each of the trials. Chicks of the vaccinated groups were all inoculated intra-abdominally on the day of hatch with a dosage of 500 plaque forming units (PFU) in trials 1,2 , and one group for trial 3; the other group for trial 3 was inoculated with commercial dosages as recommended by each of the vaccine manufacturers. No commercial dosage is established for ADOL HVT. A similar dosage was administrated following the commercial HVT manufacturer's recommendation. All experimental birds of the vaccinated and unvaccinated treatment groups were challenged with $500 \mathrm{PFU}$ of the $\mathrm{vv}+\mathrm{MDV}, 648 \mathrm{~A}$, on day 5 post hatch. In each trial, a small group of chicks, neither vaccinated nor MDV challenged, was housed along with the challenged groups as the negative control. The number of birds used for each treatment group in all three trials is given in Table $\mathbf{1}$.

\subsection{Pathological Classification}

Chickens that died during or were euthanized at the end 
Table 1. Number of chickens tested in each line and vaccine group per trial ${ }^{\mathbf{A}}$.

\begin{tabular}{|c|c|c|c|c|c|}
\hline \multirow[b]{2}{*}{ Line } & \multirow[b]{2}{*}{ Vaccine } & \multirow{2}{*}{$\begin{array}{c}\text { Trial } 1 \\
(500 \\
\text { PFU })\end{array}$} & \multirow{2}{*}{$\begin{array}{c}\text { Trial } 2 \\
(500 \\
\text { PFU })\end{array}$} & \multicolumn{2}{|c|}{ Trial 3} \\
\hline & & & & $\begin{array}{l}(500 \\
\text { PFU) }\end{array}$ & $\begin{array}{l}\text { (Comm. } \\
\text { dosage) }\end{array}$ \\
\hline \multirow[t]{7}{*}{ Line $6_{3}$} & $\operatorname{rMd} 5 \Delta \mathrm{Meq}$ & 9 & 20 & - & - \\
\hline & CVI998/Rispens 1 & 8 & 20 & 35 & 27 \\
\hline & CVI998/Rispens 2 & - & - & 35 & 30 \\
\hline & ADOL HVT & 11 & 26 & 37 & 29 \\
\hline & Commercial HVT & - & - & - & 28 \\
\hline & Unvaccinated $^{\mathrm{B}}$ & 7 & 19 & 34 & - \\
\hline & Negative Control & 1 & 7 & 8 & - \\
\hline \multirow[t]{7}{*}{ Line $7_{2}$} & $\mathrm{rMd} 5 \Delta \mathrm{Meq}$ & 8 & 24 & - & - \\
\hline & CVI998/Rispens 1 & 8 & 20 & 44 & 30 \\
\hline & CVI998/Rispens 2 & - & - & 44 & 29 \\
\hline & ADOL HVT & 9 & 21 & 40 & 30 \\
\hline & Commercial HVT & - & - & - & 30 \\
\hline & Unvaccinated $^{\mathrm{B}}$ & 9 & 29 & 51 & - \\
\hline & Negative Control & 2 & 8 & 15 & - \\
\hline \multirow[t]{5}{*}{$15 \mathrm{I}_{5} \times 7_{1}$} & CVI998/Rispens 1 & - & - & 35 & - \\
\hline & CVI998/Rispens 2 & - & - & 33 & - \\
\hline & ADOL HVT & - & - & 32 & - \\
\hline & Unvaccinated & - & - & 31 & - \\
\hline & Total & 72 & 194 & 474 & 233 \\
\hline
\end{tabular}

${ }^{\mathrm{A}}$ Comm. Dosage: commercial dosage; -: not done or not applicable; ${ }^{\mathrm{B}} \mathrm{The}$ $500 \mathrm{PFU}$ and commercial dosage treatment groups were conducted simultaneously along with one unvaccinated but challenged group in trial 3 .

of the trials were examined for gross MD lesions, which include enlarged peripheral nerves and visceral lymphomas. Histological analysis was performed on tissues of chickens with non-definitive gross lesions. Both bursa and thymus were examined for atrophy. All experimental birds were pathologically categorized either as MD or normal according to necropsy records. MD was defined as any experimental birds with an MD lesion including a gross tumor, histologically-confirmed micro-tumors, nerve enlargement(s), or died between the period of 8 days post MDV challenge and prior to experimental termination. Normal chickens were those that survived MDV challenge throughout the entire experimental period and were free of any gross MD lesion. Both bursa and thymus atrophy conditions were scored with subjective scores of 0 , 1,2 , or 3 corresponding to no, mild, moderate, or severe atrophy.

\subsection{Immunohistochemistry Analysis}

Spleen tissues from 16 vaccinated and infected line $6_{3}$ and $7_{2}$ survivors were sampled at termination of the trials. The spleen tissues were embedded in optimal cutting temperature compound (Sakura Finetek USA, Inc., Tor- rance, CA), frozen in liquid nitrogen, and stored at $-80^{\circ} \mathrm{C}$ until subsequent analysis. Immunochemistry analysis was conducted following similar procedures as described by Gimeno et al. in 2001 [29]. Briefly, the Vectastain ${ }^{\circledR}$ avidin-biotin-peroxidase complex (ABC) kit (Vector Laboratories, Burlingame, CA) was used following the manufacturer's instruction. Viral antigens were evaluated by counting positive Meq antigens in 30 randomly high power fields per sample section under $40 \times$ magnification using an Olympus BX51TF fluorescence research microscope (Olympus Co. Ltd., Japan). Means and standard errors of the positive Meq antigens were calculated by vaccinal treatment group within each genetic line of chickens.

\subsection{Statistical Analysis}

Unlike continuous traits, such as body weight that is commonly analyzed with Analysis of Variance (ANOVA), $\mathrm{MD}$ is a binary disease phenotype (MD versus normal) and, therefore, it was analyzed with a nominal logistic model to statistically determine the main effects of experimental trials (for trials 1 and 2 only), genetic line of chicken (lines $6_{3}, 7_{2}$ and $15 \mathrm{I}_{5} \times 7_{1}$ ), vaccine treatment (rMd5 $\Delta$ Meq, CVI988/Rispens 1, ADOL HVT, and unvaccinated for trials 1 and 2, and CVI988/Rispens1, CVI988/Rispens 2, ADOL HVT and commercial HVT and unvaccinated for trial 3), and all possible combinations of interactions among the main effects (full models). The logistic model fitted the nominal Y variable, MD in this study, to a linear model of the main effects and interactions.

The Whole Model Test, which is analogous to the ANOVA table, provides statistic and $p$ value for the models [31]. Likelihood Ratio Tests were used to determine the statistical significance of each main effect and effect of interactions. The main effect of trial for trials 1 and 2 was not statistically significant $(p>0.05)$ and was then removed from the model. A final reduced two-factorial logistic model including line, vaccine, and a line by vaccine interaction was used in the analyses of data from trials 1 and 2. Trial 3 data were also analyzed with the same reduced model, but the 500 PFU dosage group and the commercial dosage group were analyzed separately.

Since the main effect of trial for trials 1 and 2 was not statistically significant, pooled data of trials 1 and 2 were analyzed and presented. The statistical significance for all pairwise comparisons of MD was based on the Likelihood Ratio Tests (Tables $\mathbf{2}$ and 3). Pairwise comparisons of percentages of bursa and thymus atrophy (Tables 4-7) between line and vaccine treatment groups were tested using the z-statistic [32] with Bonferroni corrections. The estimate of protective index (PI) is a function of MD 

of Marek's Disease Virus in White Leghorn Chickens

Table 2. MD incidence and protective index for chickens of trials 1 and 2 , which were challenged with $\mathrm{vv}+\mathrm{MDV}(648 \mathrm{~A})^{\mathrm{A}}$.

\begin{tabular}{|c|c|c|c|c|c|c|c|}
\hline \multirow{2}{*}{ Line } & \multirow{2}{*}{ Vaccine } & \multicolumn{2}{|c|}{ Trial 1} & \multicolumn{2}{|c|}{ Trial 2} & \multicolumn{2}{|c|}{ Summary } \\
\hline & & $\mathrm{MD} \% \pm \mathrm{SE}$ & PI & $\mathrm{MD} \% \pm \mathrm{SE}$ & PI & $\mathrm{MD} \% \pm \mathrm{SE}^{\mathrm{B}}$ & PI \\
\hline \multirow[t]{4}{*}{ Line $6_{3}$} & $\mathrm{rMd} 5 \Delta \mathrm{Meq}$ & $22 \pm 13.9$ & 74 & $10 \pm 6.7$ & 89 & $14 \pm 6.4^{c}$ & 85 \\
\hline & CVI998/Rispens 1 & $75 \pm 15.3$ & 13 & $50 \pm 11.2$ & 47 & $57 \pm 9.4^{\mathrm{b}}$ & 38 \\
\hline & ADOL HVT & $36 \pm 14.5$ & 58 & $23 \pm 8.3$ & 76 & $27 \pm 7.3^{\mathrm{c}}$ & 71 \\
\hline & Unvaccinated & $86 \pm 13.2$ & 0 & $95 \pm 5.1$ & 0 & $92 \pm 5.2^{\mathrm{a}}$ & 0 \\
\hline \multirow[t]{4}{*}{ Line $7_{2}$} & $\mathrm{rMd} 5 \Delta \mathrm{Meq}$ & $50 \pm 17.7$ & 50 & $63 \pm 9.9$ & 38 & $59 \pm 5.2^{\mathrm{b}}$ & 41 \\
\hline & CVI998/Rispens 1 & $50 \pm 17.7$ & 50 & $70 \pm 10.2$ & 30 & $64 \pm 9.1^{b}$ & 36 \\
\hline & ADOL HVT & $100 \pm 0$ & 0 & $100 \pm 0$ & 0 & $100 \pm 0^{\mathrm{a}}$ & 0 \\
\hline & Unvaccinated & $100 \pm 0$ & 0 & $100 \pm 0$ & 0 & $100 \pm 0^{\mathrm{a}}$ & 0 \\
\hline
\end{tabular}

${ }^{\mathrm{A}} \mathrm{MD}$ : Marek's disease; SE: standard error; PI: protective index; ${ }^{\mathrm{B}} \mathrm{MD} \%$ within the column not sharing a common superscript letter are significantly different from each other $(p<0.05)$.

Table 3. MD incidence and protective index for chickens of trial 3, which were vaccinated with 500 PFU or commercially recommended dosage followed with $\mathrm{vv}+\mathrm{MDV}(648 \mathrm{~A})$ challenge ${ }^{\mathrm{A}}$.

\begin{tabular}{|c|c|c|c|c|c|}
\hline \multirow{3}{*}{ Line } & \multirow{3}{*}{ Vaccine } & \multicolumn{4}{|c|}{ Vaccination Dosage } \\
\hline & & \multicolumn{2}{|c|}{$500 \mathrm{PFU}$} & \multicolumn{2}{|c|}{ Commercial Dosage } \\
\hline & & $\mathrm{MD} \% \pm \mathrm{SE}^{\mathrm{C}}$ & PI (\%) & $\mathrm{MD} \% \pm \mathrm{SE}^{\mathrm{C}}$ & PI (\%) \\
\hline \multirow[t]{5}{*}{ Line $6_{3}$} & CVI998/Rispens 1 & $40 \pm 8.3^{\mathrm{d}}$ & 59 & $33 \pm 9.0^{c}$ & 66 \\
\hline & CVI998/Rispens 2 & $37 \pm 8.2^{\mathrm{d}}$ & 62 & $27 \pm 8.1^{\mathrm{c}}$ & 72 \\
\hline & ADOL HVT & $49 \pm 8.2^{\mathrm{cd}}$ & 49 & $17 \pm 7.0^{\mathrm{c}}$ & 82 \\
\hline & Commercial HVT & - & - & $14 \pm 6.6^{\mathrm{c}}$ & 86 \\
\hline & Unvaccinated $^{\mathrm{B}}$ & $97 \pm 2.9^{\mathrm{a}}$ & 0 & $97 \pm 2.9^{\mathrm{ab}}$ & 0 \\
\hline \multirow[t]{5}{*}{ Line $7_{2}$} & CVI998/Rispens 1 & $77 \pm 6.3^{\mathrm{bc}}$ & 23 & $73 \pm 8.1^{\mathrm{b}}$ & 27 \\
\hline & CVI998/Rispens 2 & $68 \pm 7.0^{\mathrm{b}}$ & 32 & $31 \pm 8.6^{\mathrm{c}}$ & 69 \\
\hline & ADOL HVT & $93 \pm 4.0^{\mathrm{a}}$ & 7 & $100 \pm 0.0^{\mathrm{a}}$ & 0 \\
\hline & Commercial HVT & - & - & $90 \pm 5.5^{\mathrm{ab}}$ & 10 \\
\hline & Unvaccinated $^{\mathrm{B}}$ & $100 \pm 0.0^{\mathrm{a}}$ & 0 & $100 \pm 0.0^{\mathrm{a}}$ & 0 \\
\hline \multirow[t]{4}{*}{$15 \mathrm{I}_{5} \times 7_{1}$} & CVI998/Rispens 1 & $40 \pm 8.3^{\mathrm{d}}$ & 60 & - & - \\
\hline & CVI998/Rispens 2 & $27 \pm 7.7^{\mathrm{d}}$ & 73 & - & - \\
\hline & ADOL HVT & $50 \pm 8.8^{\mathrm{cd}}$ & 50 & - & - \\
\hline & Unvaccinated & $100 \pm 0.0^{\mathrm{a}}$ & 0 & - & - \\
\hline
\end{tabular}

${ }^{\mathrm{A}} \mathrm{MD}$ : Marek's disease; SE: standard error; PI: protective index; -: not done or not applicable; ${ }^{\mathrm{B}}$ The 500 PFU and commercial dosage treatment groups were conducted simultaneously along with one unvaccinated but challenged group; ${ }^{\mathrm{C}} \mathrm{MD} \%$ not sharing a common superscript letter are significantly different from each other $(p<0.05)$.

Table 4. Percentages of vaccinated or unvaccinated chickens free of or with severe bursa atrophy induced by vv+MDV in trials 1 and 2.

\begin{tabular}{|c|c|c|c|c|c|c|c|}
\hline \multirow{2}{*}{ Line } & \multirow{2}{*}{ Vaccine } & \multicolumn{2}{|c|}{ Trial 1} & \multicolumn{2}{|c|}{ Trial 2} & \multicolumn{2}{|c|}{ Summary } \\
\hline & & $\mathrm{No} \% \pm \mathrm{SE}(\%)$ & Severe \pm SE $(\%)$ & $\mathrm{No} \% \pm \mathrm{SE}(\%)$ & Severe \pm SE $(\%)$ & $\mathrm{No} \% \pm \mathrm{SE}^{\mathrm{A}}(\%)$ & Severe $\pm \mathrm{SE}^{\mathrm{A}}(\%)$ \\
\hline \multirow[t]{4}{*}{ Line $6_{3}$} & $\mathrm{rMd} 5 \Delta \mathrm{Meq}$ & $78 \pm 13.9$ & $22 \pm 13.9$ & $85 \pm 8.0$ & $10 \pm 6.7$ & $83 \pm 7.0^{\mathrm{a}}$ & $14 \pm 6.4^{\mathrm{d}}$ \\
\hline & CVI998/Rispens 1 & $13 \pm 11.7$ & $63 \pm 17.1$ & $35 \pm 10.7$ & $40 \pm 11$ & $29 \pm 8.5^{\mathrm{cd}}$ & $46 \pm 9.4^{\mathrm{bc}}$ \\
\hline & ADOL HVT & $55 \pm 15.0$ & $27 \pm 13.4$ & $73 \pm 8.7$ & $15 \pm 7.1$ & $68 \pm 7.7^{\mathrm{ab}}$ & $19 \pm 6.4^{\mathrm{d}}$ \\
\hline & Unvaccinated & $43 \pm 18.7$ & $57 \pm 18.7$ & $16 \pm 8.4$ & $79 \pm 9.4$ & $23 \pm 8.3^{\text {cd }}$ & $78 \pm 8.7^{\mathrm{ab}}$ \\
\hline \multirow[t]{4}{*}{ Line $7_{2}$} & $\operatorname{rMd} 5 \Delta \mathrm{Meq}$ & $63 \pm 17.1$ & $38 \pm 17.1$ & $33 \pm 9.6$ & $29 \pm 9.3$ & $41 \pm 8.7^{\mathrm{bc}}$ & $31 \pm 8.2^{\mathrm{cd}}$ \\
\hline & CVI998/Rispens 1 & $50 \pm 17.7$ & $38 \pm 17.1$ & $40 \pm 11.0$ & $55 \pm 11.1$ & $43 \pm 9.4^{\mathrm{bc}}$ & $50 \pm 9.4^{\mathrm{bc}}$ \\
\hline & ADOL HVT & $0 \pm 0$ & $100 \pm 0$ & $10 \pm 6.4$ & $57 \pm 10.8$ & $7 \pm 4.6^{\mathrm{de}}$ & $70 \pm 8.6^{\mathrm{ab}}$ \\
\hline & Unvaccinated & $0 \pm 0$ & $100 \pm 0$ & $0 \pm 0$ & $83 \pm 7.0$ & $0 \pm 0^{\mathrm{e}}$ & $87 \pm 5.5^{\mathrm{a}}$ \\
\hline
\end{tabular}

No: free of bursa atrophy; Severe: severe bursa atrophy; SE: standard error; ${ }^{\mathrm{A}}$ Percentages within a column not sharing a common superscript letter were significantly different from one another $(p<0.05$ after Bonferroni adjustment). 
Table 5. Percentage of vaccinated and unvaccinated chickens free of or with severe bursa atrophy induced by $\mathrm{vv}+\mathrm{MDV}$ in trial 3.

\begin{tabular}{|c|c|c|c|c|c|}
\hline \multirow{3}{*}{ Line } & \multirow{3}{*}{ Vaccine } & \multicolumn{4}{|c|}{ Vaccination Dosage } \\
\hline & & \multicolumn{2}{|c|}{$500 \mathrm{PFU}$} & \multicolumn{2}{|c|}{ Commercial Dosage } \\
\hline & & No \pm SE $(\%)^{\mathrm{A}}$ & Severe \pm SE $(\%)^{A}$ & $\mathrm{No} \pm \mathrm{SE}(\%)^{\mathrm{A}}$ & Severe \pm SE $(\%)^{A}$ \\
\hline \multirow[t]{5}{*}{ Line $6_{3}$} & CVI998/Rispens 1 & $37 \pm 8.2^{\mathrm{bcd}}$ & $37 \pm 8.2^{\mathrm{e}}$ & $59 \pm 9.5^{\mathrm{abc}}$ & $26 \pm 8.4^{\mathrm{c}}$ \\
\hline & CVI998/Rispens 2 & $46 \pm 8.4^{\mathrm{abc}}$ & $37 \pm 8.2^{\mathrm{e}}$ & $50 \pm 9.1^{\mathrm{c}}$ & $17 \pm 6.9^{c}$ \\
\hline & ADOL HVT & $49 \pm 8.2^{\mathrm{abc}}$ & $43 \pm 8.1^{\mathrm{de}}$ & $38 \pm 9.0^{\text {bcd }}$ & $14 \pm 6.4^{\mathrm{c}}$ \\
\hline & Commercial HVT & - & - & $68 \pm 8.8^{\mathrm{ab}}$ & $14 \pm 6.6^{\mathrm{c}}$ \\
\hline & Unvaccinated & $9 \pm 4.9^{\mathrm{efg}}$ & $91 \pm 4.9^{\mathrm{ab}}$ & $9 \pm 4.9^{\mathrm{de}}$ & $91 \pm 4.9^{\mathrm{a}}$ \\
\hline \multirow[t]{5}{*}{ Line $7_{2}$} & CVI998/Rispens 1 & $25 \pm 6.5^{\text {cde }}$ & $70 \pm 6.9^{\mathrm{bcd}}$ & $30 \pm 8.4^{\mathrm{cd}}$ & $57 \pm 9.0^{b}$ \\
\hline & CVI998/Rispens 2 & $36 \pm 7.2^{\mathrm{bcd}}$ & $55 \pm 7.5^{\text {cde }}$ & $79 \pm 7.6^{\mathrm{a}}$ & $17 \pm 7.0^{\mathrm{c}}$ \\
\hline & ADOL HVT & $15 \pm 5.6^{\mathrm{def}}$ & $73 \pm 7.0^{\mathrm{bc}}$ & $3 \pm 3.1^{\mathrm{e}}$ & $77 \pm 7.7^{\mathrm{b}}$ \\
\hline & Commercial HVT & - & - & $13 \pm 6.1^{\mathrm{de}}$ & $77 \pm 7.7^{\mathrm{b}}$ \\
\hline & Unvaccinated & $4 \pm 2.7^{\mathrm{fg}}$ & $96 \pm 2.7^{\mathrm{a}}$ & $4 \pm 2.7^{\mathrm{e}}$ & $96 \pm 2.7^{\mathrm{a}}$ \\
\hline \multirow[t]{4}{*}{$15 \mathrm{I}_{5} \times 7_{1}$} & CVI998/Rispens 1 & $63 \pm 8.2^{\mathrm{ab}}$ & $31 \pm 7.8^{\mathrm{e}}$ & - & - \\
\hline & CVI998/Rispens 2 & $80 \pm 8.0^{\mathrm{a}}$ & $27 \pm 7.7^{\mathrm{e}}$ & - & - \\
\hline & ADOL HVT & $56 \pm 8.8^{\mathrm{ab}}$ & $28 \pm 7.9^{\mathrm{e}}$ & - & - \\
\hline & Unvaccinated & $0 \pm 0^{\mathrm{a}}$ & $97 \pm 3.1^{\mathrm{a}}$ & - & - \\
\hline
\end{tabular}

No: no bursa atrophy; Severe: severe bursa atrophy; SE: standard error; ${ }^{A}$ Percentages within a column not sharing a common superscript letter were significantly different from one another ( $p<0.05$ after Bonferroni adjustment).

Table 6. Percentages of vaccinated or unvaccinated chickens free of or with severe thymus atrophy induced by $\mathrm{vv}+\mathrm{MDV}$ in trials 1 and 2.

\begin{tabular}{|c|c|c|c|c|c|c|c|}
\hline \multirow{2}{*}{ Line } & \multirow{2}{*}{ Vaccine } & \multicolumn{2}{|c|}{ Trial 1} & \multicolumn{2}{|c|}{ Trial 2} & \multicolumn{2}{|c|}{ Summary } \\
\hline & & $\mathrm{No} \% \pm \mathrm{SE}(\%)$ & Severe \pm SE $(\%)$ & No $\% \pm$ SE $(\%)$ & Severe \pm SE $(\%)$ & $\mathrm{No} \% \pm \mathrm{SE}^{\mathrm{A}}(\%)$ & Severe $\pm \mathrm{SE}^{\mathrm{A}}(\%)$ \\
\hline \multirow[t]{4}{*}{ Line $6_{3}$} & rMd5 $5 \Delta \mathrm{Meq}$ & $78 \pm 13.9$ & $22 \pm 13.9$ & $85 \pm 8.0$ & $10 \pm 6.7$ & $83 \pm 7.0^{\mathrm{a}}$ & $14 \pm 6.4^{\mathrm{e}}$ \\
\hline & CVI998/Rispens 1 & $13 \pm 11.7$ & $75 \pm 15.3$ & $35 \pm 10.7$ & $45 \pm 11.1$ & $29 \pm 8.5^{\text {bd }}$ & $54 \pm 9.4^{\mathrm{bc}}$ \\
\hline & ADOL HVT & $46 \pm 15.0$ & $36 \pm 14.5$ & $73 \pm 8.7$ & $15 \pm 7.1$ & $65 \pm 7.8^{\mathrm{ac}}$ & $22 \pm 6.8^{\mathrm{de}}$ \\
\hline & Unvaccinated & $29 \pm 17.1$ & $57 \pm 18.7$ & $11 \pm 7.0$ & $90 \pm 7.0$ & $15 \pm 7.1^{\mathrm{bc}}$ & $81 \pm 7.7^{\mathrm{ab}}$ \\
\hline \multirow[t]{4}{*}{ Line $7_{2}$} & $\mathrm{rMd} 5 \Delta \mathrm{Meq}$ & $63 \pm 17.1$ & $38 \pm 17.1$ & $33 \pm 9.6$ & $25 \pm 8.8$ & $41 \pm 8.7^{\mathrm{bc}}$ & $28 \pm 7.9^{\text {cde }}$ \\
\hline & CVI998/Rispens 1 & $50 \pm 17.7$ & $25 \pm 15.3$ & $40 \pm 11.0$ & $60 \pm 11.0$ & $43 \pm 9.4^{\mathrm{bc}}$ & $50 \pm 9.4^{\mathrm{cd}}$ \\
\hline & ADOL HVT & $0 \pm 0$ & $100 \pm 0$ & $10 \pm 6.4$ & $86 \pm 7.6$ & $7 \pm 4.6^{\mathrm{de}}$ & $90 \pm 5.5^{\mathrm{a}}$ \\
\hline & Unvaccinated & $0 \pm 0$ & $100 \pm 0$ & $3 \pm 3.4$ & $83 \pm 7.0$ & $3 \pm 2.6^{\mathrm{e}}$ & $87 \pm 5.5^{\mathrm{a}}$ \\
\hline
\end{tabular}

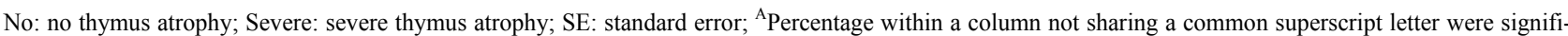
cantly different from one another ( $p<0.05$ after Bonferroni adjustment).

percentages of the unvaccinated and vaccinated groups as described in our early report [17] and lacks known statistical distribution. Therefore it is non-testable (Tables 2 and 3).

Chicken survived days were analyzed using a proportional hazards model including chicken line, vaccine, and line by vaccine interaction. The differences among the trends of survival day patterns of chicken line and vaccine treatment group were tested with Log-Rank and Wilcoxon tests. All statistical analyses were performed using Statistical Analysis Software JMP version 8.01 and SAS version 9.2 (SAS Institute Inc., NC, USA).

\section{Results}

MD incidence and vaccinal protective indices were sum- marized in Table 2 for trials 1 and 2 and Table 3 for trial 3.

\subsection{Genetic Resistance of Lines $6_{3}, 7_{2}$, and $15 I_{5} \times 7_{1}$ to MD upon vv+MDV, 648A, Challenge}

The vv+MDV, 648A, challenge without prior vaccination resulted in MD incidence ranging from $86 \%$ to $97 \%$ in line $6_{3}$ and $100 \%$ in line $7_{2}$ in trials $1-3$, and $100 \%$ in the $15 \mathrm{I}_{5} \times 7_{1}$ chickens in trial 3 (Tables 2 and 3 ). In pooled data of all three trials, the MD incidences of the unvaccinated line $6_{3}(95 \%)$ and line $7_{2}(100 \%)$ groups were significantly different from each other $(p<0.05)$; the MD incidence of line $6_{3}$ or line $7_{2}$ chickens was not significantly different from that of the $15 \mathrm{I}_{5} \times 7_{1}$ chickens $(p>$ $0.05)$. 
Table 7. Percentage of vaccinated or unvaccinated chickens free of or with severe thymus atrophy induced by vv+MDV in trial 3.

\begin{tabular}{|c|c|c|c|c|c|}
\hline \multirow{3}{*}{ Line } & \multirow{3}{*}{ Vaccine } & \multicolumn{4}{|c|}{ Vaccination Dosage } \\
\hline & & \multicolumn{2}{|c|}{$500 \mathrm{PFU}$} & \multicolumn{2}{|c|}{ Commercial Dosage } \\
\hline & & $\mathrm{No} \pm \mathrm{SE}(\%)^{\mathrm{A}}$ & Severe \pm SE $(\%)^{\mathrm{A}}$ & $\mathrm{No} \pm \mathrm{SE}(\%)^{\mathrm{A}}$ & Severe \pm SE $(\%)^{\mathrm{A}}$ \\
\hline \multirow[t]{5}{*}{ Line $6_{3}$} & CVI998/Rispens 1 & $43 \pm 8.4^{\mathrm{abc}}$ & $31 \pm 7.8^{\mathrm{e}}$ & $63 \pm 9.3^{\mathrm{ab}}$ & $26 \pm 8.4^{\mathrm{b}}$ \\
\hline & CVI998/Rispens 2 & $46 \pm 8.4^{\mathrm{abc}}$ & $37 \pm 8.2^{\mathrm{de}}$ & $53 \pm 9.1^{\mathrm{abc}}$ & $20 \pm 7.3^{b}$ \\
\hline & ADOL HVT & $51 \pm 8.2^{\mathrm{abc}}$ & $43 \pm 8.1^{\mathrm{dc}}$ & $38 \pm 9.0^{\mathrm{bc}}$ & $17 \pm 7.0^{\mathrm{b}}$ \\
\hline & Commercial HVT & - & - & $68 \pm 8.8^{\mathrm{ab}}$ & $18 \pm 7.3^{\mathrm{b}}$ \\
\hline & Unvaccinated & $9 \pm 4.9^{\mathrm{ef}}$ & $91 \pm 4.9^{\mathrm{ab}}$ & $9 \pm 4.9^{\mathrm{de}}$ & $91 \pm 4.9^{\mathrm{a}}$ \\
\hline \multirow[t]{5}{*}{ Line $7_{2}$} & CVI998/Rispens 1 & $25 \pm 6.5^{\mathrm{cde}}$ & $75 \pm 6.5^{\mathrm{bc}}$ & $27 \pm 8.1^{\mathrm{cd}}$ & $67 \pm 8.6^{\mathrm{a}}$ \\
\hline & CVI998/Rispens 2 & $34 \pm 7.1^{\mathrm{bcd}}$ & $64 \pm 7.2^{\mathrm{cd}}$ & $76 \pm 7.9^{\mathrm{a}}$ & $17 \pm 7.0^{\mathrm{b}}$ \\
\hline & ADOL HVT & $13 \pm 5.3^{\mathrm{def}}$ & $78 \pm 6.5^{\mathrm{bc}}$ & $3 \pm 3.1^{\mathrm{e}}$ & $90 \pm 5.5^{\mathrm{a}}$ \\
\hline & Commercial HVT & - & - & $10 \pm 5.5^{\mathrm{de}}$ & $73 \pm 8.1^{\mathrm{a}}$ \\
\hline & Unvaccinated & $6 \pm 3.3^{\mathrm{ef}}$ & $94 \pm 3.3^{\mathrm{ab}}$ & $6 \pm 3.3^{\mathrm{de}}$ & $94 \pm 3.3^{\mathrm{a}}$ \\
\hline \multirow[t]{4}{*}{$15 \mathrm{I}_{5} \times 7_{1}$} & CVI998/Rispens 1 & $60 \pm 8.3^{\mathrm{ab}}$ & $34 \pm 8.0^{\mathrm{e}}$ & - & - \\
\hline & CVI998/Rispens 2 & $70 \pm 8.0^{\mathrm{a}}$ & $27 \pm 7.7^{\mathrm{e}}$ & - & - \\
\hline & ADOL HVT & $53 \pm 8.8^{\mathrm{abc}}$ & $38 \pm 8.6^{\mathrm{de}}$ & - & - \\
\hline & Unvaccinated & $0 \pm 0^{\mathrm{f}}$ & $97 \pm 3.1^{\mathrm{a}}$ & - & - \\
\hline
\end{tabular}

No: no thymus atrophy; Severe: severe thymus atrophy; SE: standard error; ${ }^{A}$ Percentages within a column not sharing a common superscript letter were significantly different from one another ( $p<0.05$ after Bonferroni adjustment).

\section{2. rMd5 $\Delta$ Meq Protective Efficacy}

The rMd5 $\Delta$ Meq conveyed comparable protection compared to ADOL HVT but significantly higher protection than CVI988/Rispens in lines $6_{3}$ in trials 1 and 2 . The rMd5 $\Delta$ Meq and CVI988/Rispens conveyed comparable protections, which were significantly higher than ADOL HVT did in line $7_{2}$ in trials 1 and $2(p<0.05)$. In pooled data of trials 1 and 2, the MD incidences were $14 \%$ and $59 \%$ for the rMd5 $\Delta \Delta$ Meq vaccinated lines $6_{3}$ and $7_{2}$ chickens, respectively (Table 2).

\subsection{CVI988/Rispens Protective Efficacy}

At the dosage of $500 \mathrm{PFU}$, the MD incidences of CVI988/Rispens 1 vaccinated line $6_{3}$ chickens ranged from 40 to $75 \%$ with protective indices of $13 \%$ up to $59 \%$ in trials 1-3; CVI988/Rispens 2 was comparable to CVI988/Rispens 1 in protection of line $6_{3}$ in trial 3 . The MD incidences of CVI988/Rispens 1 vaccinated line $7_{2}$ chickens ranged from $50 \%$ to $77 \%$ with protective indices of $23 \%$ to $50 \%$ in trials $1-3$. The CVI988/Rispens 2 was also comparable to CVI988/Rispens 1 in protection of line $7_{2}$ in trial 3 (Tables 2 and 3). The CVI988/Rispens 1 and Rispens 2 conveyed comparable protection for the $15 \mathrm{I}_{5} \times 7_{1}$ chickens as did for line $6_{3}$, which were significantly better than the protection conveyed for line $7_{2}$ (Table 3).

\subsection{HVT Protective Efficacy}

At a 500 PFU dosage, the MD incidences of ADOL HVT vaccinated line $6_{3}$ chickens ranged from $23 \%$ to $49 \%$ with protective indices of $49 \%$ to $76 \%$ in trials $1-3$. The MD incidences of ADOL HVT vaccinated line $7_{2}$ chickens ranged from $93 \%$ to $100 \%$ with protective index of $0 \%$ to $7 \%$ in trials $1-3$ (Tables 2 and 3 ). ADOL HVT provided comparable protection as did $\mathrm{rMd} 5 \Delta \mathrm{Meq}$ and significantly better protection than CVI988/Rispens 1 to line $6_{3}$ chickens but no protection to line $7_{2}$ chickens in trials 1 and 2 (Table 2). ADOL HVT provided comparable protection to $15 \mathrm{I}_{5} \times 7_{1}$ chickens as did both CVI988/Rispens 1 and 2 in trial 3 (Table 3 ).

\subsection{Protective Efficacy at Commercially Recommended Dosages}

Under commercially recommended dosages, CVI988/Rispens 1, CVI988/Rispens 2, ADOL and commercial HVT conveyed comparable protection to line $6_{3}$ but varied protections to line $7_{2}$ chickens in Trial 3. The CVI988/Rispens 2 conveyed significantly better protection than CVI988/ Rispens 1 , ADOL and commercial HVT to line $7_{2}$ chickens (Table 3).

\subsection{The Effect of Chicken Line, Vaccine, and Line by Vaccine Interaction on Protective Efficacy}

The Log Linear model analysis suggested chicken line, vaccine, and line by vaccine interaction all had very significant effect on MD incidences in trials 1 and 2 and 
also in trial 3 of the commercial dosage groups based on the Effect Likelihood Ratio tests $(p<0.05)$. However, the chicken line by vaccine interaction effect on MD incidence was not statistically significant in trial 3 chickens that were vaccinated with a 500 PFU dosage $(p>0.05)$.

\subsection{Survival Days Varied between Chicken Lines and Vaccine Group}

Chicken line and vaccine significantly affected survived day patterns based on Log-Rank and Wilcoxon Tests. In pooled data of trials 1 and 2 , both $\mathrm{rMd} 5 \Delta \mathrm{Meq}$ and $\mathrm{ADOL}$ HVT vaccinated line $6_{3}$ chickens survived significantly longer than the CVI988/Rispens 1 vaccinated group. The rMd5 $\Delta$ Meq and CVI988/Rispens 1 vaccinated line $7_{2}$ groups, however, survived significantly longer than the ADOL HVT vaccine group. All vaccinated groups survived significantly longer than the unvaccinated group within each line (Figure 1, upper panel, $p<0.01$ ).

At commercial dosages, all of the vaccinated groups of line $6_{3}$ and the CVI988/Rispens 2 vaccinated line $7_{2}$ chickens had comparable survival patterns, which survived significantly longer than those of the CVI988/ Rispens 1, ADOL HVT or commercial HVT vaccinated line $7_{2}$ groups. All vaccinated groups were significantly different from the unvaccinated groups of line $6_{3}$ and $7_{2}$, and the line $6_{3}$ unvaccinated was significantly different from line $7_{2}$ in survived day pattern (Figure 1, lower panel, $p<0.01$ ).

Chicken line by vaccine interaction also significantly affected the survived days in trials 1 and 2 and in trial 3 chickens vaccinated with commercial dosages based on the Effect Likelihood Ratio Tests $(p<0.01)$. The line by vaccine interaction effect on survived days of the trial 3 chickens vaccinated with a 500 PFU dosage was not statistically significant $(p>0.05)$.

\subsection{Chicken Line and Vaccine Affected both Bursa and Thymus Atrophy}

Most of the line $6_{3}$ chickens vaccinated with $\mathrm{rMd} 5 \Delta \mathrm{Meq}$ or ADOL HVT were free of bursa atrophy $(83 \%$ and $68 \%$, respectively) and the percentages were significantly higher than the CVI988/Rispens 1 vaccinated or unvaccinated line $6_{3}$ groups in trials 1 and 2 . The $\mathrm{rMd} 5 \Delta \mathrm{Meq}$ and CVI988/Rispens 1 vaccinated line $7_{2}$ chickens that were free of bursa atrophy ( $41 \%$ and $43 \%$, respectively), however, were comparable to each other $(p>0.05)$ but significantly different from the ADOL HVT vaccinated and unvaccinated line $7_{2}$ groups $(7 \%$ and $0 \%$, respectively; $p<0.05$ ). The percentages of chickens that suffered from severe bursa atrophy in trials 1 and 2 ranked from high to low as unvaccinated $\geq$ CVI988/Rispens $1>$ ADOL HVT $\geq \operatorname{rMd} 5 \Delta$ Meq in line $6_{3}$, whereas in line $7_{2}$, as unvaccinated $\geq$ ADOL HVT $\geq$ CVI988/Rispens $1 \geq$ rMd5 $\Delta$ Meq (Table 4).

In trial 3 of the 500 PFU groups, more CVI988/Rispens 1 and ADOL HVT vaccinated line $7_{2}$ chickens suffered from severe bursa atrophy than CVI988/Rispens 1 and ADOL HVT vaccinated $15 \mathrm{I}_{5} \times 7_{1}$ and CVI988/Rispens 1 vaccinated line $6_{3}$ chickens $(p<0.05)$. The percentages of unvaccinated chickens that suffered from severe bursa atrophy were comparable among the line $6_{3}$, $7_{2}$ and $15 \mathrm{I}_{5} \times 7_{1}$ group $(p>0.05)$.

Among the commercial dosage groups, CVI988/Rispens 2 vaccinated line $7_{2}$ group was significantly lower in percentage of chickens suffered from severe bursa atrophy (17\%) than the CVI988/Rispens 1 (57\%), ADOL and commercial HVT (77\%) vaccinated line $7_{2}$ groups ( $p$ $<0.05)$ (Table 5).

The percentages of thymus atrophy for trials 1 and 2 and trial 3 are given in Tables 6 and 7, respectively. The relative percentages of chickens free of or that suffered from severe thymus atrophy between lines and among the vaccinated groups were highly comparable to the percentages of bursa atrophy data as described above.

\subsection{The rMd5 $\Delta M e q$ and CVI988/Rispens Vaccinated Chickens Free of MDV Meq Antigen Expression at Eight Weeks Post MDV Infection}

The immunohistochemistry analysis data showed the rMd5 $\Delta$ Meq or CVI988/Rispens 1 vaccinated chickens from both line $6_{3}$ and $7_{2}$ were free of MDV Meq antigen expression at eight weeks post MDV infection. Two out of three line $6_{3}$ chickens vaccinated with HVT and two out of two unvaccinated line $6_{3}$ chickens, however, were positive for the MDV Meq antigen. The average number of positive MDV Meq antigen per random high power field for HVT vaccinated and unvaccinated line $6_{3}$ chickens were estimated as $1.88 \pm 0.45$ and $3.47 \pm 0.72$, respectively. No HVT vaccinated or unvaccinated line $7_{2}$ infected chickens survived at the time of the sampling for this analysis.

\section{Discussion and Conclusions}

Innate resistance to MD of genetic lines of chickens is of critical importance to vaccinal protective efficacy [17]. In this study, the percentages of MD of the unvaccinated line $6_{3}, 7_{2}$, and $15 I_{5} \times 7_{1}$ chickens were not significantly different from each other $(p>0.05)$ due to the high virulence of the challenge virus, $\mathrm{vv}+648 \mathrm{~A}$, which overwhelmed the innate resistance and adaptive immunity of these three lines of chickens and therefore, masked the underlying difference in innate resistance between the lines of chickens. The survival days of the line $6_{3}$, how- 

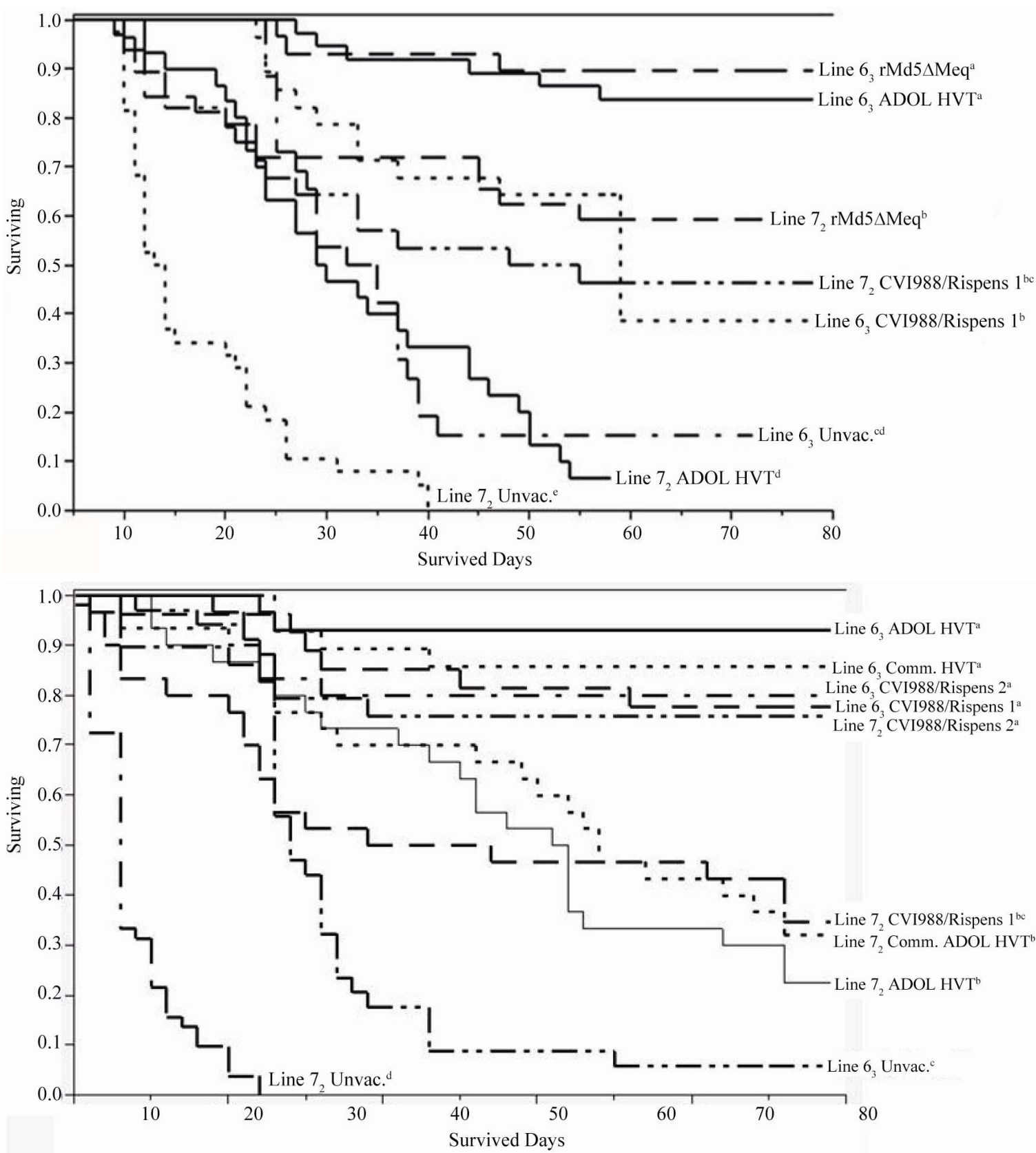

Figure 1. Survival plots of pooled survival day data (trials 1 and 2) showing 1) rMd5 $\triangle$ Meq and ADOL HVT conveyed comparable and the longest survival trends in line $6_{3}$ chickens among all the vaccinated groups of both lines; 2) Followed by the rMd5 $\Delta$ Meq vaccinated line $7_{2}$ and CVI988/Ripens vaccinated line $6_{3}$ and line $7_{2}$ chickens (comparable trends to one another); 3) Then the ADOL HVT vaccinated line $7_{2}$ and the unvaccinated line $6_{3}$ chickens sharing comparable and poor survival trends, but still survived significantly longer than the unvaccinated line $7_{2}$ group (upper panel); 4) With commercially recommended dosages (trial 3), both CVI988/Rispens and both ADOL and commercial HVT vaccinated line $6_{3}$, and CVI988/Rispens 2 vaccinated line $7_{2}$ chickens survived longer than the CVI988/Rispens 1, ADOL and commercial HVT vaccinated $7_{2}$ chickens; unvaccinated line $6_{3}$ and line $7_{2}$ groups also differed in survival days from each other (lower panel).

ever, were significantly different from those of line $7_{2}$ in all trials $(p<0.01)$ indicating that the line $6_{3}$ is indeed relatively more resistant to $\mathrm{MD}$ than line $7_{2}$, which is in good agreement with other reports [19,23,27].

All vaccines provided greater protection to the line $6_{3}$ chickens than to the counterparts of the line $7_{2}$ except CVI988/Rispens 1 in trials 1 and 2 at 500 PFU dosage and CVI988/Rispens 2 in trial 3 at commercial dosage in terms of MD incidences (Tables $\mathbf{2}$ and $\mathbf{3}$ ), survival days (Figure 1), and bursa thymus atrophy (BTA) (Tables 
4-7). The differential protective efficacy should be primarily attributable to the non- $M H C$ genetics that underlies the difference in innate resistance to MD between the lines $6_{3}$ and 72 . This finding confirms an earlier report [17] that non- $M H C$ host genetics plays an important role in modulating vaccine protective efficacy, and echoes with a study, in which significant variation in response to HIV vaccination among individual human as well as among human populations were observed [33].

Furthermore, the CVI988/Rispens 1 vaccinated line $6_{3}$ chickens had significantly higher MD incidence than the ADOL HVT vaccinated line $6_{3}$ chickens but the CVI988/ Rispens 1 vaccinated line $7_{2}$ chickens had significantly lower MD than ADOL HVT vaccinated line $7_{2}$ chickens in trials 1 and 2 (Table 2). This phenomenon was also observed in survival days and the numbers of vaccinated chickens free of BTA in trials 1 and 2 (Figure 1, Tables 4 and 6). The reversed orders in ranking between CVI988/ Rispens 1 and ADOL HVT vaccinated line $6_{3}$ chickens and CVI988/Rispens 1 and ADOL HVT vaccinated line $7_{2}$ chickens suggested an interaction between the genetic lines of chickens and vaccines. This interaction should represent an interaction between the non- $M H C$ host genetics of the chicken lines and the genomes of the two vaccine viruses. The Effect Likelihood Ratio Tests indicated that the line by vaccine interaction significantly affected MD incidences as well as survival days in trials 1 and 2 and in the commercial dosage groups of trial 3 ( $p$ $<0.01)$.

The $15 \mathrm{I}_{5} \times 7_{1} \mathrm{~F}_{1}$ hybrid chicks were included in trial 3 since this kind of chicks have been the choice for research on MDV and vaccine trials at ADOL for a long time. Line $7_{1}$ is a related subline of the line $7_{2}$ with the same $M H C B^{* 2}$ haplotype. Line $15 \mathrm{I}_{5}$ has a $B^{*} 15$ haplotype and is very susceptible to MD. Thus, the hybrid $15 \mathrm{I}_{5} \times 7_{1}$ chicks carry both the line $7_{1}$ and line $15 \mathrm{I}_{5}$ genetics background and are heterozygous for $M H C, B * 2 /$ $B * 15$. The unvaccinated $15 \mathrm{I}_{5} \times 7_{1}$ chickens were as highly susceptible as the unvaccinated lines $6_{3}$ and $7_{2}$ chickens, but the vaccinated groups were almost the same as the vaccinated line $6_{3}$ groups and significantly differed from the vaccinated line $7_{2}$ groups (Tables $\mathbf{3}, \mathbf{5}$ and 7 ). The better protective efficacy in the $15 \mathrm{I}_{5} \times 7_{1}$ chickens ought to be attributable, at least partially, to the $B^{*} 15$ haplotype, since $B^{*} 2$ chickens were reportedly less well protected than $B^{*} 15$ chickens by any of the three serotypes of MD vaccines [14].

Maternal antibody significantly interferes with vaccine protective efficacy. Maternal antibodies to HVT interfere with cell-associated and cell-free HVT [34] but it is unclear whether the maternal antibodies to HVT interfere with either rMd5 $\Delta$ Meq or CVI988/Rispens. Homologous maternal antibodies to CVI988 clone C, however, cause a significant decrease in the protective efficacy of CVI988 Clone $\mathrm{C}$ vaccine against vvMDV challenge [35]. Both of the line $6_{3}$ and $7_{2}$ chickens used in trial 1 were maternal antibody positive to HVT but line $6_{3}$ and $7_{2}$ as well as the $15 \mathrm{I}_{5} \times 7_{1}$ chickens used in trials 2 and 3 were negative. Although there was a trend of numerically lower MD\% and higher PI of each vaccinated group in trial 2 compared with that of trial 1 , a summary column was presented for the pooled trial 1 and 2 data in Tables 2, 4 and 6 based on a likelihood ratio test that the MD incidence between trial 1 and trial 2 was not statistically different $(p>0.05)$ from each other, despite the difference in maternal antibody status to HVT.

MD viral antigen (Meq) was not detected in spleens by immunohistochemistry analysis in small numbers of randomly sampled survivors of both line $6_{3}$ and $7_{2}$ chickens vaccinated either with $\mathrm{rMd} 5 \Delta \mathrm{Meq}$ or CVI988/Rispens 1. The Meq, however, was detected in line $6_{3}$ chickens either vaccinated with HVT or unvaccinated suggesting that both serotype 1 vaccines, rMd5 $\Delta$ Meq and CVI988/ Rispens, are of stronger antiviral activity than the serotype 3 vaccine, HVT. This finding is in good agreement with a report by Lee et al. [36], in which a significant reduction $(100 \%, 98.4 \%)$ of viremia levels was observed in $\mathrm{rMd} 5 \Delta$ Meq or CVI988/Rispens vaccinated chickens followed by vvMDV challenge at the $7^{\text {th }}$ week post infection. Rapid depletion of infected viruses not only reduces animal mortality, but also slows or even prevents viral shedding in late stages of MDV infected chickens.

Data from this study confirms that non- $M H C$ host genetics plays an important role conferring vaccine protective efficacy against MD in chickens. More interestingly, although the protective index of all vaccine strains was higher in resistant than in susceptible line of chickens, the difference for HVT protection was striking. The data also suggests that a chicken line by vaccine interaction may modulate vaccine protection efficacy. Cui et al. [37] recently reported that challenging chickens with a recombinant field isolate of MDV, termed GX0101, or vvMDV, Md5, induced a high but comparable level of mortality. But when the same kind of chickens was HVT vaccinated followed by GX0101 or Md5 challenge, the GX0101 challenged group resulted in significantly fewer mortalities than the Md5 group $(p<0.01)$, suggesting an interaction between challenge MDV and vaccine viruses that confers vaccine protection efficacy. In this light, it is anticipated that, in addition to other known factors, vaccine protective efficacy might also be modulated by a three-way interaction between host genetic background, vaccine, and challenge viruses.

\section{Acknowledgements}

We thank Evelyn Young, Barbara Riegle, and Barry Coul- 
son for their excellent technical supports in conducting challenging experiments of this study. This work was supported in part by a research grant, RE Project \#F020, from US Poultry \& Egg Harold E. Ford Foundation.

\section{REFERENCES}

[1] B. W. Calnek, "Pathogenesis of Marek's Disease Virus Infection," Current Topics in Microbiology and Immunology, Vol. 255, 2001, pp. 25-55. doi:10.1007/978-3-642-56863-3 2

[2] B. W. Calnek, "Marek's Disease Vaccines," Developments in Biological Standardization, Vol. 52, 1982, pp. 401-405.

[3] R. L. Witter, "New Serotype 2 and Attenuated Serotype 1 Marek's Disease Vaccine Viruses: Comparative Efficacy," Avian Diseases, Vol. 31, No. 4, 1987, pp. 752-765. doi: $10.2307 / 1591027$

[4] A. F. Islam, S. W. Walkden-Brown, P. J. Groves and G. J. Underwood, "Effects of Vaccine Dose, Virus Challenge Dose and Interval from Vaccination to Challenge on Protection of Broiler Chickens against Marek's Disease Virus Challenge," Australian Veterinary Journal, Vol. 85, No. 9, 2007, pp. 348-355. doi:10.1111/j.1751-0813.2007.00195.x

[5] J. D. Blaxland, A. J. Macleod and T. Hall, "Trials with Marek's Disease Vaccines Prepared from a Turkey Herpes Virus and an Attenuated Marek's Disease Virus," Veterinary Record, Vol. 97, No. 3, 1975, pp. 50-52. doi:10.1136/vr.97.3.50

[6] H. G. Purchase and W. Okazaki, "Effect of Vaccination with Herpesvirus of Turkeys (HVT) on Horizontal Spread of Marek's Disease Herpesvirus," Avian Diseases, Vol. 15, No. 2, 1971, pp. 391-397. doi.org/10.2307/1588710

[7] L. N. Payne and K. Venugopal, "Neoplastic Diseases: Marek's Disease, Avian Leukosis and Reticuloendotheliosis," Scientific and Technical Review, Vol. 19, No. 2, 2000, pp. 544-564.

[8] B. H. Rispens, H. van Vloten, N. Mastenbroek, H. J. Maas and K. A. Schat, "Control of Marek's Disease in the Netherlands. I. Isolation of an Avirulent Marek's Disease Virus (Strain CVI 988) and Its Use in Laboratory Vaccination Trials," Avian Diseases, Vol. 16, No. 1, 1972, pp. 108-125. doi: $10.2307 / 1588905$

[9] K. A. Schat and B. W. Calnek, "Characterization of an Apparently Nononcogenic Marek's Disease Virus," Journal of the National Cancer Institute, Vol. 60, No. 5, 1978, pp. 1075-1082.

[10] R. L. Witter and B. R. Burmester, "Differential Effect of Maternal Antibodies on Efficacy of Cellular and CellFree Marek's Disease Vaccines," Avian Pathology, Vol. 8, No. 2, 1979, pp. 145-156. doi:10.1080/03079457908418337

[11] L. F. Lee, H. Zhang, M. Heidari, B. Lupiani and S. M. Reddy, "Evaluation of Factors Affecting Vaccine Efficacy of Recombinant Marek's Disease Virus Lacking the Meq Oncogene in Chickens," Avian Diseases, Vol. 55, No. 2, 2011, pp. 172-179.

\section{doi:10.1637/9575-101510-Reg.1}

[12] J. S. Gavora and J. L. Spencer, "Studies on Genetic Resistance of Chickens to Marek's Disease-A Review," Comparative Immunology, Microbiology and Infectious Diseases, Vol. 2, No. 2-3, 1979, pp. 359-371. doi:10.1016/0147-9571(79)90022-5

[13] L. D. Bacon and R. L. Witter, "Influence of B-Haplotype on the Relative Efficacy of Marek's Disease Vaccines of Different Serotypes," Avian Diseases, Vol. 37, No. 1, 1993, pp. 53-59. doi:10.2307/1591457

[14] L. D. Bacon and R. L. Witter, " $B$ Haplotype Influence on the Relative Efficacy of Marek's Disease Vaccines in Commercial Chickens," Poultry Science, Vol. 73, No. 4, 1994, pp. 481-487. doi:10.3382/ps.0730481

[15] L. D. Bacon and R. L. Witter, "Serotype Specificity of BHaplotype Influence on the Relative Efficacy of Marek's Disease Vaccines," Avian Diseases, Vol. 38, No. 1, 1994, pp. 65-71. doi:10.2307/1591838

[16] L. D. Bacon and R. L. Witter, "Efficacy of Marek's Disease Vaccines in Mhc Heterozygous Chickens: Mhc Congenic $\times$ Inbred line $F_{1}$ Matings," Journal of Heredity, Vol. 86, No. 4, 1995, pp. 269-273.

[17] S. Chang, J. R. Dunn, M. Heidari, L. F. Lee, J. Song, C. W. Ernst, Z. Ding, L. D. Bacon and H. M. Zhang, "Genetics and Vaccine Efficacy: Host Genetic Variation Affecting Marek's Disease Vaccine Efficacy in White Leghorn Chickens," Poultry Science, Vol. 89, No. 10, 2010, pp. 2083-2091. doi:10.3382/ps.2010-00740

[18] W. M. Muir, G. K. Wong, Y. Zhang, J. Wang, M. A. M. Groenen, R. P. M. A. Crooijmans, H.-J. Megens, H. M. Zhang, J. C. McKay, S. McLeod, R. Okimoto, J. E. Fulton, P. Settar, N. P. O'sullivan, A. Vereijken, A. Jungerius-Rattink, G. A. A. Albers, C. Taylor Lawley, M. E. Delany and H. H. Cheng, "Review of the Initial Validation and Characterization of a 3K Chicken SNP Array," World's Poultry Science Journal, Vol. 64, No. 2, 2008, pp. 219-225. doi:10.1017/S0043933908000019

[19] L. D. Bacon, H. D. Hunt and H. H. Cheng, "A Review of the Development of Chicken Lines to Resolve Genes Determining Resistance to Diseases," Poultry Science, Vol. 79, No. 8, 2000, pp. 1082-1093.

[20] H. M. Zhang, H. D. Hunt, G. B. Kulkarni, D. E. Palmquist and L. D. Bacon, "Lymphoid Organ Size Varies among Inbred Lines 6(3) and 7(2) and Their Thirteen Recombinant Congenic Strains of Chickens with the Same Major Histocompatibility Complex," Poultry Science, Vol. 85, No. 5, 2006, pp. 844-853.

[21] N. Yonash, L. D. Bacon and E. J. Smith, "Concentration of Immnoglobulin G in Plasma Varies among 6C.7 Recombinant Congenic Strains of Chickens," Poultry Science, Vol. 81, No. 8, 2002, pp. 1104-1108.

[22] L. D. Bacon, H. D. Hunt and H. H. Cheng, "A Review of the Development of Chicken Lines to Resolve Genes Determining Resistance to Diseases," Poultry Science, Vol. 79, No. 8, 2000, pp. 1082-1093.

[23] L. D. Bacon, H. D. Hunt and H. H. Cheng, "Genetic Resistance to Marek's Disease," Current Topics in Microbiology and Immunology, Vol. 255, 2001, pp. 121-141. 
doi:10.1007/978-3-642-56863-3 5

[24] R. L. Witter, "Increased Virulence of Marek's Disease Virus Field Isolates," Avian Diseases, Vol. 41, No. 1, 1997, pp. 149-163. doi.org/10.2307/1592455

[25] R. L. Witter, B. W. Calnek, C. Buscaglia, I. M. Gimeno and K. A. Schat, "Classification of Marek's Disease Viruses According to Pathotype: Philosophy and Methodology," Avian Pathology, Vol. 34, No. 2, 2005, pp. 75-90. doi:10.1080/03079450500059255

[26] S. M. Reddy, B. Lupiani, I. M. Gimeno, R. F. Silva, L. F. Lee and R. L. Witter, "Rescue of a Pathogenic Marek's Disease Virus with Overlapping Cosmid DNAs: Use of a pp38 Mutant to Validate the Technology for the Study of Gene Function," Proceedings of the National Academy of Sciences of USA, Vol. 99, No. 10, 2002, pp. 7054-7059. doi:10.1073/pnas.092152699

[27] R. L. Witter, L. D. Bacon, H. D. Hunt and H. H. Cheng, "Genetics and Vaccines for the Future Control of Marek's Disease," 1994, pp. 90-96. http://www.poultryscience.org/pba/1952-2003/1994/1994 $\% 20$ Witter.pdf

[28] R. L. Witter, "Marek's Disease Vaccines-Past, Present and Future (Chicken vs. Virus-A Battle of the Centuries)," In: K. A. Schat, R. W. Morgan, M. M. S. Parcells, and J. L. Spencer, Eds., Current Progress on Marek's Disease Research, American Association of Avian Pathologists, Kennett Square, 2001, pp. 1-9.

[29] I. M. Gimeno, R. L. Witter, H. D. Hunt, L. F. Lee, S. M. Reddy and U. Neumann, "Marek's Disease Virus Infection in the Brain: Virus Replication, Cellular Infiltration, and Major Histocompatibility Complex Antigen Expression," Veterinary Pathology, Vol. 38, No. 5, 2001, pp. 491-503.doi:10.1354/vp.38-5-491

[30] J. L. Liu, Y. Ye, Z. Qian, Y. Qian, D. J. Templeton, L. F. Lee and H. J. Kung, "Functional Interactions between Herpesvirus Oncoprotein MEQ and Cell Cycle Regulator
CDK2," Journal of Virology, Vol. 73, No. 5, 1999, pp. 4208-4219.

[31] SAS Institute Inc., "Logistic Regression for Niminal and Ordinal Response," Statistics and Graphics Guide, SAS Institute Inc., Cary, 2008, pp. 431-454.

[32] S. Geng, and F. J. Hills, "Biometrics in Agriculture Science," Kendall/Hunt Publishing Company, Dubuque, 1989.

[33] G. de Buy, "Cofactors That May Influence Vaccine Responses," Current Opinion in HIV \& AIDS, Vol. 5, No. 5, 2010, pp. 404-408. doi:10.1097/COH.0b013e32833d1fca

[34] S. Chang, Z. Ding, J. R. Dunn, L. F. Lee, M. Heidari, J. Song, C. W. Ernst and H. M. Zhang, "A Comparative Evaluation of the Protective Efficacy of rMd5dMeq and CVI988/Rispens against a vv+Strain of Marek's Disease Virus Infection in a Series of Recombinant Congenic Strains of White Leghorn Chickens," Avian Diseases, Vol. 55, No. 3, 2011, pp. 384-390. doi:10.1637/9524-091310-Reg.1

[35] G. F. de Boer, J. E. Groenendal, H. M. Boerrigter, G. L. Kok and J. M. Pol, "Protective Efficacy of Marek's Disease Virus (MDV) CVI-988 CEF65 Clone C against Challenge Infection with Three Very Virulent MDV Strains," Avian Diseases, Vol. 30, No. 2, 1986, pp. 276-283. doi: $10.2307 / 1590529$

[36] L. F. Lee, B. Lupiani, R. F. Silva, H. J. Kung and S. M. Reddy, "Recombinant Marek's Disease Virus (MDV) Lacking the Meq Oncogene Confers Protection against Challenge with a Very Virulent plus Strain of MDV," Vaccine, Vol. 26, No. 15, 2008, pp. 1887-1892. doi:10.1016/j.vaccine.2008.01.046

[37] Z. Cui, G. Zhuang, X. Xu, A. Sun and S. Su, "Molecular and Biological Characterization of a Marek's Disease Virus Field Strain with Reticuloendotheliosis Virus LTR Insert,"Virus Genes, Vol. 40, No. 2, 2010, pp. 236-243. doi:10.1007/s11262-009-0437-z 